\title{
A tale of two pities
}

\section{Autologous melanoma vaccines on the brink}

David Berd

DB Consultants; Wyncote, PA USA
Keywords: vaccine, autologous, melanoma, haptens, heat shock proteins

Abbreviations: BCG, Bacillus CalmetteGuerin; CTL, cytotoxic T lymphocytes; DNP, dinitrophenyl; DTH, delayed type hypersensitivity; HSP, heat shock proteins; MCA, methylcholanthrene; SC, subcutaneously; TCR, T cell receptor; TNCB, trinitrochlorobenzene; TNP, trinitrophenyl; UV, ultraviolet

Submitted: 05/22/12

Accepted: 05/30/12

http://dx.doi.org/10.4161/hv.20923

Correspondence to: David Berd; Email: davidberd@ aol.com
$\mathrm{T}$ his paper reviews and compares two autologous vaccine technologies for human melanoma that failed to obtain marketing approval after 10-15 y of clinical development-the HSP vaccine invented by Srivastava and developed by the company, Antigenics, and the hapten-modified cellular vaccine invented by Berd and developed by AVAX Technologies. Both vaccines had a strong basic science background with a well-understood mechanism of action. The HSP vaccine failed in a phase III pivotal trial, while the haptenized cellular vaccine was never adequately tested in a phase III trial because of regulatory and financial problems. It is proposed that the phase I-II clinical trials of the HSP vaccine neglected to define optimal dose, schedule and route of administration, which, together with safety, are the major reasons for doing such trials. Therefore, the phase III trial was bound to fail because it was based on insufficient immunopharmacological information. Developers of the haptenized cellular vaccine underestimated the manufacturing and regulatory hurdles inherent to that technology and were therefore unable to complete a pivotal trial. Valuable lessons can be learned by acknowledging the mistakes made in these attempts to bring forward new treatments that could have eased the burdens of melanoma patients.

For autologous cancer vaccines, it perpetually seems to be the best and worst of times - the best because the developing science always supports them, the worst because their clinical development always is fraught with problems.
This paper analyzes and compares two autologous vaccines for melanoma: (1) a vaccine composed of heat shock proteins (HSP) derived from autologous melanoma cells, ${ }^{1}$ and (2) a vaccine composed of intact melanoma cells modified with a hapten, dinitrophenyl (DNP). ${ }^{2}$ These technologies share several important strengths and weaknesses, including: (1) well-defined mechanism of action, (2) well-established preclinical models, (3) promising clinical results in early phase trials, (4) challenges in biomanufacturing and (5) failure to obtain marketing approval after 10-15 y of commercial development.

The HSP vaccine was developed in the laboratory of P.K. Srivastava at the University of Connecticut School of Medicine. It was licensed by a biotechnology company, Antigenics (now known as Agenus), under whose auspices clinical trials were initiated in 1997. The designated commercial name for the melanoma vaccine was Oncophage ${ }^{\circledR}$ (now known as Prophage $\left.{ }^{\circledR}\right)$. Although Oncophage ${ }^{\circledR}$ was based on Srivastava's HSP vaccine, the two are not identical by criteria used by the Food and Drug Administration (FDA), and so this review will distinguish between them whenever possible.

The autologous, DNP-modified melanoma vaccine was developed in our laboratory at Thomas Jefferson University, where we began phase I-II clinical trials in 1988. The technology was licensed by AVAX Technologies in 1996, which initiated its own clinical trials in 1997 under the commercial name, MVAX ${ }^{\circledR}$. As noted for Oncophage ${ }^{\circledR}$, AVAX made modifications to the manufacturing methods originally described for the autologous, 
DNP-modified vaccine, so it is reasonable to differentiate them.

\section{HSP Vaccine}

Origin and preclinical studies. The HSP vaccine had its origin in Srivastava's attempt to explicate the immunochemistry of a classic immunotherapy model-the murine methylcholanthrene (MCA) induced sarcoma. In a landmark paper, Prehn and Main ${ }^{3}$ discovered that the protective effect of immunizing mice against a sarcoma was individually tumor-specific. Mice that had been surgically cured after injection and subcutaneous growth of MCA sarcoma \#1 were in all cases resistant to re-challenge with MCA sarcoma \#1. Remarkably, they did not resist challenge with any other MCA sarcomas, even though those tumors were indistinguishable from sarcoma \#1, using the technology available at that time. The reciprocal relationship was established as well: for example, mice surgically cured of sarcoma \#2 resisted re-challenge with the same sarcoma but not with MCA-induced sarcoma \#1. Prehn and Main's work was the first to show that tumor-specific immunity could be induced in inbred mice and was the basis for all the animal and human immunotherapy experiments that followed it.

Srivastava's early studies had shown that injection of heat shock proteins derived from MCA sarcomas could result in protection from challenge against the corresponding tumor cells but not against tumor cells from a separately induced MCA sarcoma. All that remained to complete the picture was the demonstration of a structural difference between HSP of different MCA sarcomas and a difference between HSP derived from sarcomas vs. normal tissues. But this beautiful hypothesis was fated to be slain by an ugly fact (quotation attributed to Thomas Huxley, 1825-1895): no such differences could be detected. Not until it was subsequently discovered that HSP function as carriers of small peptides did it become clear that the tumor specificity and the individual tumor specificity resided in the variable structures not of the HSP but of their associated peptides. ${ }^{4,5}$ So far, it has not been possible to identify the peptides conferring specificity, but this did not prove to be an obstacle in developing HSP as tumor immunotherapy.

In a key paper, Srivastava ${ }^{1}$ demonstrated the potency of a HSP vaccine in mice bearing the Lewis Lung Tumor. This was a true immunotherapy model, since vaccine injections were initiated after the subcutaneously implanted tumors were palpable, and was clinically-relevant in that primary tumors were excised after metastasizing. Mice injected with a HSP vaccine derived from the tumor developed an average of 20 metastases per lung compared with 200 metastases per lung in those administered saline. About $80 \%$ of the tumor HSP vaccine mice were apparently cured. The minimum effective dose was $15 \mathrm{ug}, 5$ ug being ineffective. Injection of HSP derived from normal liver produced no therapeutic benefit. Prior depletion of $\mathrm{T}$ cells (either $\mathrm{CD}^{+}$or $\mathrm{CD} 8^{+}$) or NK cells abrogated the therapeutic effect. Vaccines composed of tumor-derived HSP were noted to be effective, although to a varying degree, in several other murine tumor models, including B16 melanoma, a UV-induced carcinoma, and a methylcholanthrene induced sarcoma.

Clinical studies. These remarkable results led to the licensing of the HSP vaccine to Antigenics and the initiation of clinical trials. The clinical data were positive but with variable results, and they suggested a major difference between the murine and human anti-tumor immune response to HSP vaccines. For example, in a trial of 16 patients with a variety of advanced cancers, ${ }^{6}$ one minor anti-tumor response was observed and immunologic studies showed ambiguous results. In a larger study $(\mathrm{n}=64)$ of metastatic melanoma patients ${ }^{7}$ two complete clinical responses were observed, both in patients with skin metastases. The dose was either 5 ug or 50 ug administered either intradermally or subcutaneously. This study was closely followed by one in which 38 melanoma patients were injected with autologous HSP at a dose of $25 \mathrm{ug}$ sc weekly in combination with $\alpha$ interferon and GM-CSF. ${ }^{8}$ There were no antitumor responses. In vitro immunological tests were performed in about half the patients with variable results.

This summary of the phase I-II trials of autologous HSP vaccine in metastatic melanoma may not be complete since some smaller studies were presented only in abstract form and other studies sponsored by Antigenics may not have been published at all. However, these studies seemed to form the basis of the phase III pivotal trial discussed below. At least two anti-tumor responses were documented and toxicity was shown to be minimal. However, these studies collectively failed to establish an optimal dose, schedule of administration, or route of administration for the HSP vaccine, all of which would seem to be necessary before proceeding to phase III. A major reason for this failure was the investigators' inability to establish an anti-tumor immunological response that was consistently enhanced in patients receiving HSP melanoma vaccine.

The results of the phase III study were disappointing. 9 Patients with stage IV melanoma ( $\mathrm{n}=322$ ) were randomly assigned (2:1) to receive HSP vaccine (now known as vitespen) or standard of care, i.e., physicians choice, which was usually single agent chemotherapy. Only patients randomized to vaccine underwent surgery to harvest autologous tumor tissue. Remarkably 61/215 patients assigned to vitespen received no doses of HSP vaccine because of quality control failure, usually a yield of tumor cells inadequate to prepare vaccines. By the rules of the protocol, these untreated patients in the treatment arm were included in the intentto-treat analysis.

Given this burden of clinical design impediments, it was not surprising that the study failed to demonstrate the efficacy of the HSP vaccine. The overall survivals of the treatment and control groups were indistinguishable. Following publication of this trial, Antigenics terminated the clinical development of the HSP vaccine in metastatic melanoma.

Summary. Undoubtedly, there were multiple reasons for the failure of this highly interesting and promising technology in clinical trials. A rather stimulating paper previously published by Srivastava's group $^{10}$ underscores one of them. As in previous studies, they showed that immunizing mice with HSP derived from an MCA-induced sarcoma induced protection against challenge with live sarcoma cells with complete and permanent 
rejection of tumors in most experiments. However, the result was dependent on the dose of vaccine as well on the route of injection, as shown in Figure 1, extracted from the original publication. Using the subcutaneous route a dose of 10 ug was highly effective but lower and higher doses failed. With the intradermal route, the optimal dose was 10-fold less$1.0 \mathrm{ug}$ - with negative results at higher doses. One wonders why these impressive results did not impel the designers of the clinical trials to take more care in establishing a dose-response curve in cancerbearing patients.

\section{DNP-Modified Melanoma Cells}

Origin and preclinical studies. Haptens were discovered by Karl Landsteiner, who used them to explore the breadth and fine sensitivity of antibody responses. Landsteiner ${ }^{11}$ worked with a variety of simple haptens, including nitrophenyls and phenyl arsonates, that were incapable of inducing an immune response by themselves, but became immunogenic when they were attached covalently to a protein carrier. He coined the term hapten from the Greek haptein, meaning to fasten.

Landsteiner made what was at the time an astounding observation: Rabbits immunized with a haptenized protein produced antibodies to a new antigenic determinant on hapten-protein conjugate. Weigle extended these observations by showing that hapten conjugation could break natural tolerance. ${ }^{12}$ The injection of rabbits with homologous thyroglobulin in incomplete Freund's adjuvant produced, as expected, little or no antibody to thyroglobulin. However, rabbits injected with thyroglobulin that had been modified with arsanilic acid plus sulfanilic acid or with TNP produced precipitating antibody to both modified and native thyroglobulin. Moreover, some of the rabbits developed histological evidence of autoimmune thyroiditis. Once the animals had been immunized, the height of their antibody titers and the severity of the thyroiditis could be increased by administering booster injections of native thyroglobulin.

What are the new antigenic determinants produced by hapten conjugation

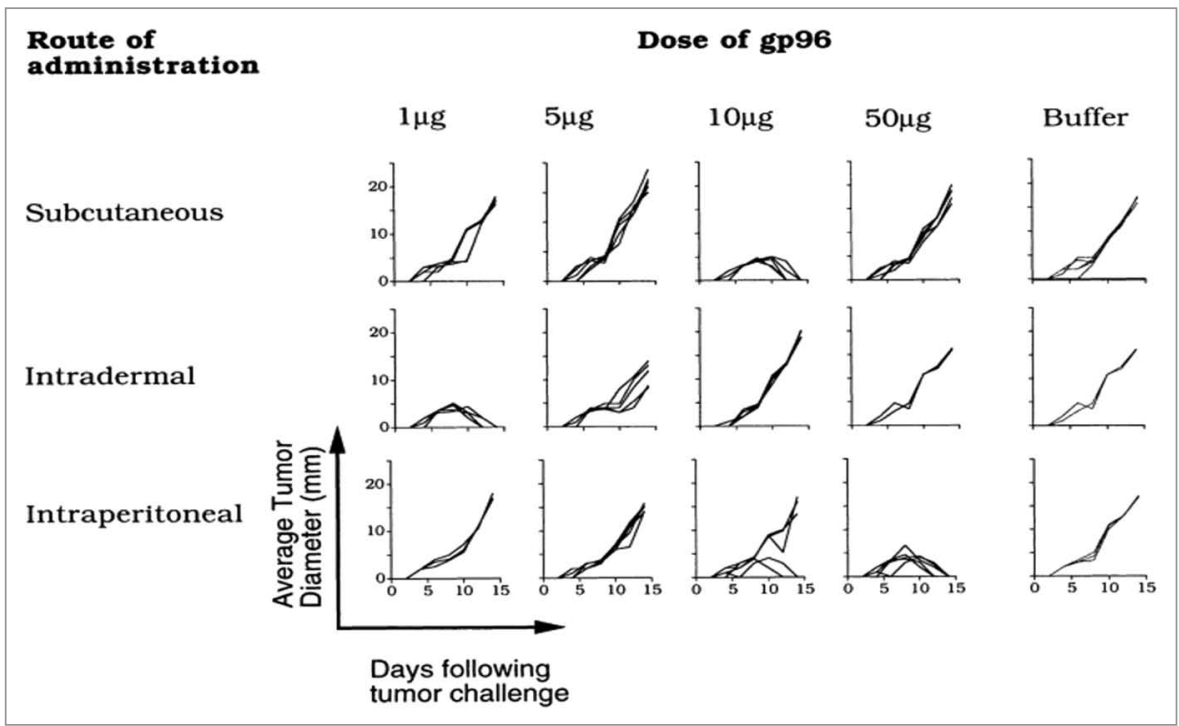

Figure 1. Dose restriction of immunogenicity of tumor-derived gp96. BALB/c mice were immunized with Meth A gp96 in the quantities and routes indicated. Immunizations were carried out twice per week, and the doses indicated represent the quantities administered at each immunization. All mice were challenged with 100,000 Meth A cells 1 week after the last immunization. Each line shows the kinetics of tumor growth in a single mouse. Reproduction with permission, Figure 1 from Chandawarkar, et al. ${ }^{10}$

that must have excited and perplexed the early investigators of hapten immunology? An impressive body of work by H.U. Weltzien's group appears to have solved the mystery. ${ }^{13}$ By immunizing mice to TNP-modified syngeneic spleen cells, they obtained cytotoxic $\mathrm{T}$ cells that lysed the modified spleen cells in an H2-restricted manner. The cytotoxic $\mathrm{T}$ cells also recognized TNP-modified peptides extracted from tryptic digests of TNP-modified bovine serum albumin. Subsequently, these investigators synthesized a variety of class I MHC-binding peptides that contained TNP-lysine at various positions. Cytotoxic $\mathrm{T}$ cell clones were tested for ability to lyse target cells that had bound one of the synthetic peptides.

They demonstrated the following: $\mathrm{T}$ cells recognized the $\mathrm{MHC}$-associated TNP-modified peptides. Hapten modification of the MHC complex itself was of minimal significance. The vast majority of CTL clones responded to multiple H2-binding peptides that had in common a TNP-lysine in position 4 and, for these, the recognition was largely independent of the amino acid sequence. However, a minor fraction of TNP-specific $\mathrm{T}$ cell clones recognized only certain sequences of TNP-modified peptides, and those $\mathrm{T}$ cell clones also recognized unmodified peptides; i.e., there was associative recognition of unmodified peptides by $\mathrm{T}$ cell clones generated by immunization with haptenmodified peptides.

The authors explained these results by postulating self-reactive $\mathrm{T}$ cells that survive thymic selection because they have low affinity for self peptides. Haptenization then increased the binding of TCR to self peptide enough for $\mathrm{T}$ cell activation and, once activated, the $T$ cells could react with unmodified peptide.

Fujiwara et al. ${ }^{14}$ applied this approach to the treatment of murine tumors. They found that a fairly large $(8 \mathrm{~mm}$ diameter) plasmacytoma could be made to regress completely by intratumoral injection of TNCB, producing a kind of in situ haptenization with TNP. As a result, these animals became resistant to challenge with unmodified tumor cells. This immunotherapy was particularly interesting because it worked not only with a transplantable tumor, but also with an autochthonous tumor that was induced in the mice by inoculation of methylcholanthrene.

The most recent publication on the use of hapten modification for experimental immunotherapy is from Sojka et al. ${ }^{15}$ 
They used the highly metastatic 410.4 tumor that had originated from a spontaneous murine mammary carcinoma. The tumor was injected into the mammary fat pad and was allowed to grow to $6-8 \mathrm{~mm}$ diameter and then excised. Following surgery mice were treated with multiple injections of a vaccine consisting of irradiated tumor cells haptenized with DNP and then mixed with BCG. Low dose cyclophosphamide was administered three days prior to each vaccine injection. Control mice received the identical treatment regimen except that the tumor cells in the vaccine were irradiated but not hapten modified. These experimental conditions were designed to mimic the post-surgical adjuvant protocols frequently used in clinical vaccine studies and, specifically, to reproduce experimentally the observations in melanoma patients, which are described below.

The result was positive and highly reproducible: mice that received DNPmodified vaccine had significantly longer relapse-free survival than animals receiving the unmodified vaccine, which, incidentally, was no better than saline. The protective effect of the haptenized vaccine was dependent on both $\mathrm{CD}^{+}$and $\mathrm{CD}^{+}$ T cells. Moreover, both gamma interferon and tumor necrosis factor were essential, since in vivo depletion of either with a monoclonal antibody abrogated the protective effect.

Clinical studies-Thomas Jefferson University. Clinical trials of this technology were begun by our group at Thomas Jefferson University in $1988 .{ }^{16}$ The method of preparation of the vaccine eventually became a major regulatory issue, so it needs to be described briefly. Metastatic tumor was excised, maintained at $4^{\circ}$, and delivered to the laboratory within $48 \mathrm{~h}$ of excision. Tumor cells were extracted by enzymatic dissociation with collagenase, aliquotted, frozen in a controlled rate freezer, and stored in liquid nitrogen until needed. On the day that a patient was to be treated, an aliquot of cells was thawed, washed and irradiated to 2,500 cGy. Then the cells were washed again and modified with DNP for $30 \mathrm{~min}$ by the method of Miller and Claman. ${ }^{17}$ After washing, the cells were counted, suspended in $0.2 \mathrm{ml}$ Hanks solution with human albumin, and maintained at $4^{\circ}$ until administered, usually within one hour.

Two adjuvants supplemented the vaccine: (1) BCG, which was added to the vaccine just before administration in an amount designed to produce a mild inflammatory papule at the injection site. (2) Low dose cyclophosphamide was administered three days before the first dose of vaccine because of its known immunopotentiating properties. ${ }^{18,19}$ The DNP-vaccine was always administered intradermally, the preferred method to yield an optimal $\mathrm{T}$ cell response. ${ }^{20}$ Determining the optimal vaccine dose was the subject of several phase I-II studies ${ }^{21}$ with the development of DTH to autologous, unmodified melanoma cells as the endpoint. There was no significant association between the magnitude of DTH and the number of live (trypanblue-excluding) melanoma cells administered per dose over a dosage range of $0.5-25.0 \times 10^{6}$. Although all vaccine contained variable numbers of dead cells as well, the proportion of dead cells did not affect the immunogenicity, and in fact, slightly increased it.

In contrast, the schedule of vaccine administration was discovered to be critically important to its effectiveness. Of the five schedules tested, the DTH responses induced by two of them were significantly inferior to those induced by the other three. ${ }^{22}$ This result was not due to inadvertent patient selection, and the schedules did not affect the magnitude of the DTH responses to PPD. Retrospective analysis indicated that effective schedules had incorporated an induction vaccine dose administered 5-7 d before cyclophosphamide. Patients who had received an optimally-timed induction dose had significantly greater DTH responses to unmodified melanoma cells and longer survival times than patients who received the induction dose at the wrong time. ${ }^{22}$

An important observation that was sustained over the course of these studies is the association of a positive DTH response to unmodified, autologous melanoma with prolonged survival. The survival of stage IV patients who developed a positive DTH to unmodified tumor cells was significantly longer than the survival of those who did not: 16.5 mo vs. 8.4 mo, respectively ( $\mathrm{p}=$ 0.023 , log rank test). ${ }^{23}$ In stage III patients treated in the adjuvant setting, the development of a positive response to unmodified tumor cells was associated with significantly greater 5-y survival (59.3\% vs. 29.3\%, p < $0.001, \log$ rank test). ${ }^{22}$

Finally, the clinical results of phase III trials appeared promising: In a series of studies with 83 evaluable patients with stage IV melanoma, there were 11 responses: 2 complete, 4 partial and 5 mixed; 2 patients were judged to have stable disease. ${ }^{23}$ Both complete responses and two of the four partial responses occurred in patients with lung metastases. Response durations were as follows: partial responses-5, 6, 8 and 47+ months; complete responses-12, 29 mo. In 214 stage III patients $(97$ = stage IIIB, $117=$ stage IIIC) treated in the post-surgical adjuvant setting, the five-year overall survival was $44 \%$, which appears to compare favorably with reported surgical survival rates of $20-25 \% .^{24}$

Clinical studies-AVAX technologies. When AVAX began its clinical studies of what became known as MVAX $^{\circledR}$ in 1997, all of the necessary data seemed to be in place to ensure a strong phase III pivotal trial. The vaccine was safe. Detailed dosage-schedule data were available, and a robust intermediate endpoint, DTH to unmodified melanoma cells, had been identified. Since the haptenized vaccine appeared most effective in patients with low tumor burdens, the first pivotal trial was a post-surgical adjuvant study in patients with stage IIIB and IIIC melanoma. Subjects were randomized at a $2: 1$ ratio to receive $\mathrm{MVAX}^{\circledR}$ or high dose $\alpha$ interferon, the treatment of choice at that time. ${ }^{25}$ There was every reason to expect that MVAX $^{\circledR}$ could be proposed for marketing approval by 2002 .

As they have been saying in sad stories from time immemorial, it was not to be! The shelf life of what was soon called the fresh vaccine was too short to allow for completion of quality control tests, particularly sterility testing. A significant proportion of vaccines were eventually found to be bacterially contaminated. Interestingly, the most common contaminant was the anaerobic skin contaminant, Propionibacterium acnes. How melanoma specimens acquire this organism is an interesting story in itself, but not relevant 


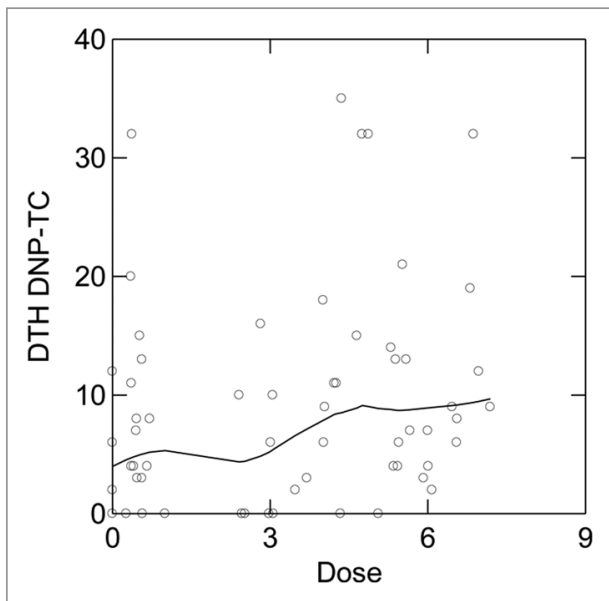

Figure 2. Dose-response curve of frozen format of MVAX ${ }^{\circ}$. Abscissa: Dose of MVAX administered, as measured by number of melanoma cells per dose. Ordinate: Intensity of DTH to DNP-modified autologous melanoma cells as measured by maximum diameter of induration in $\mathrm{mm}$. Although four dosage levels were defined, the actual dose received by a patient assigned to a given dosage level was allowed to vary within a specified range. Squared multiple $R=0.453 p<0.001$. This linear regression analysis indicates a significant direct relationship between the dose of MVAX administered and the post-treatment DTH response.

for telling in this context. After the accrual of about $10 \%$ of the planned subjects, FDA stopped the trial. Overnight, a small but prospering company with a strong technology was on the verge of extinction.

AVAX survived, but only after a significant modification in the manufacturing procedures that had seemed so well-established. A frozen version of MVAX $^{\circledR}$ was developed by combining the tumor dissociation and vaccine production and freezing the final product, giving it a shelf life measured in months rather than hours. All quality control tests could be completed before each batch of vaccine was released; batches that were not sterile were discarded. In some important ways, frozen $\mathrm{MVAX}^{\circledR}$ was identical to the haptenized vaccine produced in the laboratory at Thomas Jefferson. It consisted of intact, irradiated melanoma cells modified by the hapten, DNP. However, there was one significant difference: in frozen MVAX $^{\circledR}$, all of the melanoma cells were dead as defined by uptake of vital dyes.

Although previous studies of the fresh vaccine suggested that dead (but intact by flow cytometric analysis) melanoma cells were immunogenic, ${ }^{21}$ the frozen version of MVAX $^{\circledR}$ was considered by FDA to be a new product requiring a new phase I trial. The study was conducted in 82 patients with stage IV melanoma with tumor tissue that could be resected for preparation of vaccine. ${ }^{26}$ They were assigned to one of four vaccine dosage levels depending on the yield of tumor cells obtained: $5 \times 10^{6}$, $2.5 \times 10^{6}, 0.5 \times 10^{6}$ and 0 (diluent only). The treatment schedule was the optimal schedule as defined by studies described above: Day 1: vaccine \#1 injected id without adjuvant; Day 7: cyclophosphamide $300 \mathrm{mg} / \mathrm{m}^{2}$; Days 10, 17, 24, 31, 38 and 45: vaccine injected id after admixture with the adjuvant BCG. DTH responses to autologous tumor cells, both DNPmodified and unmodified, were tested $2-3$ weeks before and $2-3$ weeks after the course of vaccine administration; positive DTH was defined as at least $5 \mathrm{~mm}$ diameter of induration.

The 64 evaluable patients met the following criteria: (1) vaccines passed quality control; (2) baseline DTH testing negative; and (3) received both pre- and postvaccine DTH testing. Statistical analysis of each dosage level was performed using the Simon two-stage design. ${ }^{27}$ Only the high dose arm $\left(5 \times 10^{6}\right.$ melanoma cells per dose) was clearly positive in the original analysis. After a pre-planned extension of that arm, 23/30 (77\%) of those patients developed (+) DTH to autologous DNPmodified melanoma cells. Moreover, 9/16 tested (56\%) developed (+) DTH to autologous unmodified melanoma cells.
These results are indistinguishable from the DTH results obtained with fresh vaccine cells in previous studies. Of the three lower dosage arms, two were negative and one was equivocal by predetermined criteria. As shown in Figure 2, there was a significant direct relationship between frozen $\mathrm{MVAX}^{\circledR}$ dose and the intensity of post-vaccine DTH to autologous, DNPmodified melanoma cells. There were no safety issues with frozen MVAX ${ }^{\circledR}$.

So, by 2008, AVAX was ready for a phase III pivotal trial of frozen MVAX ${ }^{\circledR}$ and received a Special Protocol Assessment from FDA to support the study. This time the target population was stage IV melanoma, randomized to receive $\mathrm{MVAX}^{\circledR}$ + adjuvants (BCG, cyclophosphamide, low dose IL2) or placebo + adjuvants; the major endpoints were tumor regression and overall survival. The study design was controversial, although well-grounded, ${ }^{28}$ but there is little point to further discussion of it, since the study died a premature death because of insufficient funding.

\section{Conclusions}

So, two autologous vaccine technologies, two good ideas, stumbled on the bumpy path to marketing approval. Were they failures? Perhaps they were, but not scientific failures. No new science has arisen that invalidates the ideas upon which these technologies rest. Autologous vaccines still make sense, and they fit into the new, although not so novel, concept of personalized cancer therapy. Increasingly sophisticated molecular analyses have only verified what Prehn knew in 1957that there may be important differences between tumors that have the same histology and even the same cause. The rationale for allogeneic vaccines, never very strong, is even shakier now. The idea that each patient's tumor is antigenically distinct, shocking in 1957 and annoying in 1997, now is very hard to resist.

Nevertheless, distinction between the overlapping stories of the autologous HSP vaccine and the haptenized cellular vaccine must be made if we are to learn anything going forward. MVAX ran aground because of the manufacturing and regulatory problems it engendered. It was never adequately tested in 
a randomized trial. Conflict of interest aside, the phase I-II clinical data and the supporting immunological work still look convincing. The HSP melanoma vaccine did fail in a randomized trial. Moreover, as this paper argues, the randomized trial was conducted before the developers knew enough about the immunopharmacology to design a trial properly. Of the many phase I-II trials, none properly addressed dose, schedule of administration or route of administration, possibly because no intermediate end point for measuring these parameters was ever identified. Antigenics seems to have ignored its own preclinical literature, which clearly showed that these parameters can determine success or failure.

What of the future? Both biotechnology companies still exist, although Antigenics felt a need to change its name to Agenus. AVAX has had the spirit to remain AVAX. Unfortunately, neither company is any longer actively pursuing a melanoma vaccine, each having turned its attention to other cancer types. That is a severe blow to the hopes of melanoma patients, who are still in dire need of effective, non-toxic treatments, the latest spectacular developments notwithstanding. ${ }^{29}$ The only good news for them is that the mistakes of the past are clear. It would be far, far better not to repeat them.

\section{Acknowledgements}

The authors wishes to acknowledge AVAX Technologies for allowing use of the data generated in it phase I-II trial of MVAX ${ }^{\circledR}$. In addition, he is grateful to the hundreds of melanoma patients who consent to participate in the haptenized vaccine studies. The author received no financial assistance in the preparation of this manuscript.

\section{References}

1. Tamura Y, Peng P, Liu K, Daou M, Srivastava PK. Immunotherapy of tumors with autologous tumorderived heat shock protein preparations. Science 1997; 278:117-20; PMID:9311915; http://dx.doi. org $/ 10.1126 /$ science.278.5335.117.

2. Berd D. Hapten-Modified Vaccines. In Morse MA, Clay TM, Lyerly HK, (Eds): Handbook of Cancer Vaccines. Totowa NJ, Humana Press 2004; 225-48.

3. Prehn RT, Main JM. Immunity to methylcholanthrene-induced sarcomas. J Natl Cancer Inst 1957; 18:769-78; PMID:13502695.

4. Srivastava PK, Heike M. Tumor-specific immunogenicity of stress-induced proteins: convergence of two evolutionary pathways of antigen presentation? [Review]. SeminImmunol 1991; 3:57-64; PMID:1893123.
5. Binder RJ, Kelly JB, 3rd, Vatner RE, Srivastava PK. Specific immunogenicity of heat shock protein gp 96 derives from chaperoned antigenic peptides and not from contaminating proteins. J Immunol 2007; 179:7254-61; PMID:18025167.

6. Janetzki S, Palla D, Rosenhauer V, Lochs H, Lewis JJ, Srivastava PK. Immunization of cancer patients with autologous cancer-derived heat shock protein gp96 preparations: a pilot study. Int J Cancer 2000; 88:232-8; PMID:11004674; http://dx.doi. org/10.1002/1097-0215(20001015)88:2<232::AIDIJC14>3.0.CO;2-8.

7. Belli F, Testori A, Rivoltini L, Maio M, Andreola G, Sertoli MR, et al. Vaccination of metastatic melanoma patients with autologous tumor-derived heat shock protein gp96-peptide complexes: clinical and immunologic findings. J Clin Oncol 2002; 20:416980; PMID:12377960; http://dx.doi.org/10.1200/ JCO.2002.09.134.

8. Pilla L, Patuzzo R, Rivoltini L, Maio M, Pennacchioli E, Lamaj E, et al. A phase II trial of vaccination with autologous, tumor-derived heat-shock protein peptide complexes Gp96, in combination with GM-CSF and interferon-alpha in metastatic melanoma patients. Cancer Immunol Immunother 2006; 55:958-68; PMID:16215718; http://dx.doi. org/10.1007/s00262-005-0084-8.

9. Testori A, Richards J, Whitman E, Mann GB, Lutzky J, Camacho L, et al.; C-100-21 Study Group. Phase III comparison of vitespen, an autologous tumorderived heat shock protein gp96 peptide complex vaccine, with physician's choice of treatment for stage IV melanoma: the C-100-21 Study Group. J Clin Oncol 2008; 26:955-62; PMID:18281670; http:// dx.doi.org/10.1200/JCO.2007.11.9941.

10. Chandawarkar RY, Wagh MS, Srivastava PK. The dual nature of specific immunological activity of tumor-derived gp96 preparations. J Exp Med 1999; 189:1437-42; PMID:10224283; http://dx.doi. org/10.1084/jem.189.9.1437.

11. Landsteiner K. Artificial conjugated antigens. Serological reactions with simple chemical compounds, in The Specificity of Serological Reactions. New York, Dover Publications, Inc. 1962; 156-209.

12. Weigle WO. The production of thyroiditis and antibody following injection of unaltered thyroglobulin without adjuvant into rabbits previously stimulated with altered thyroglobulin. J Exp Med 1965; 122:1049-62; PMID:5297361; http://dx.doi. org/10.1084/jem.122.6.1049.

13. Martin S, Weltzien HU. T cell recognition of haptens, a molecular view. Int Arch Allergy Immuno 1994; 104:10-6; PMID:7524836; http://dx.doi org/10.1159/000236703.

14. Fujiwara H, Moriyama Y, Suda T, Tsuchida T, Shearer GM, Hamaoka T. Enhanced TNP-reactive helper $\mathrm{T}$ cell activity and its utilization in the induction of amplified tumor immunity that results in tumor regression. J Immunol 1984; 132:1571-7; PMID:6198397.

15. Sojka DK, Felnerova D, Mokyr MB. Anti-metastatic activity of hapten-modified autologous tumor cell vaccine in an animal tumor model. Cancer Immunol Immunother 2002; 51:200-8; PMID:12012107; http://dx.doi.org/10.1007/s00262-002-0271-9.

16. Berd D, Murphy G, Maguire HC Jr, Mastrangelo MJ. Immunization with haptenized, autologous tumor cells induces inflammation of human melanoma metastases. Cancer Res 1991; 51:2731-4; PMID:2021952.

17. Miller SD, Claman HN. The induction of haptenspecific $\mathrm{T}$ cell tolerance by using hapten-modified lymphoid cells. I. Characteristics of tolerance induction. J Immunol 1976; 117:1519-26; PMID:1087316.
18. Maguire HC Jr, Ettore VL. Enhancement of dinitrochlorobenzene (DNCB) contact sensitization by cyclophosphamide in the guinea pig. J Invest Dermatol 1967; 48:39-43; PMID:6018241.

19. Berd D, Maguire HC Jr, Mastrangelo MJ Potentiation of human cell-mediated and humoral immunity by low-dose cyclophosphamide. Cancer Res 1984; 44:5439-43; PMID:6488195.

20. Kenney RT, Frech SA, Muenz LR, Villar CP, Glenn GM. Dose sparing with intradermal injection of influenza vaccine. N Engl J Med 2004; 351:2295301; PMID:15525714; http://dx.doi.org/10.1056/ NEJMoa043540

21. Berd D, Sato T, Mastrangelo MJ. Effect of the dose and composition of an autologous hapten-modified melanoma vaccine on the development of delayedtype hypersensitivity responses. Cancer Immunol Immunother 2002; 51:320-6; PMID:12111120; http://dx.doi.org/10.1007/s00262-002-0285-3.

22. Berd D, Sato T, Maguire HC Jr, Kairys J, Mastrangelo MJ. Immunopharmacologic analysis of an autologous, hapten-modified human melanoma vaccine. J Clin Oncol 2004; 22:403-15; PMID:14691123; http://dx.doi.org/10.1200/JCO.2004.06.043.

23. Berd D, Sato T, Cohn H, Maguire HC Jr, Mastrangelo MJ. Treatment of metastatic melanoma with autologous, hapten-modified melanoma vaccine: regression of pulmonary metastases. Int J Cancer 2001; 94:531 9; PMID:11745440; http://dx.doi.org/10.1002/ ijc.1506.abs.

24. Coit DG, Rogatko A, Brennan MF. Prognostic factors in patients with melanoma metastatic to axillary or inguinal lymph nodes.A multivariate analysis. Ann Surg 1991; 214:627-36; PMID:1953117; http:// dx.doi.org/10.1097/00000658-199111000-00014.

25. Kirkwood JM, Strawderman MH, Ernstoff MS, Smith TJ, Borden EC, Blum RH. Interferon alfa$2 \mathrm{~b}$ adjuvant therapy of high-risk resected cutaneous melanoma: the eastern Cooperative Oncology Group Trial EST 1684. J Clin Oncol 1996; 14:7-17; PMID:8558223.

26. Berd D, Bloome E, Schea H. Dose-response relationship of autologous, hapten-modified vaccine in metastatic melanoma. Proc Am Assoc Cancer Res 2007; 48:630.

27. Simon R. Optimal two-stage designs for phase II clinical trials. Control Clin Trials 1989; 10:1-10; PMID:2702835; http://dx.doi.org/10.1016/01972456(89)90015-9.

28. Lotem M, Shiloni E, Pappo I, Drize O, Hamburger $\mathrm{T}$, Weitzen $\mathrm{R}$, et al. Interleukin-2 improves tumour response to DNP-modified autologous vaccine for the treatment of metastatic malignant melanoma. $\mathrm{B}_{\mathrm{r}}$ J Cancer 2004; 90:773-80; PMID:14970852; http:// dx.doi.org/10.1038/sj.bjc.6601563.

29. Chapman PB, Hauschild A, Robert C, Haanen JB, Ascierto P, Larkin J, et al.; BRIM-3 Study Group. Improved survival with vemurafenib in melanoma with BRAF V600E mutation. N Engl J Med 2011; 364:2507-16; PMID:21639808; http://dx.doi. org/10.1056/NEJMoa1103782. 